\title{
Diagnosis of Observations after Fit of Multivariate Skew $t$-Distribution: Identification of Outliers and Edge Observations from Asymmetric Data
}

\author{
Seung-Gu Kim ${ }^{1}$ \\ ${ }^{1}$ Department of Data and Information, Sangji University \\ (Received October 22, 2012; Revised November 5, 2012; Accepted November 8, 2012)
}

\begin{abstract}
This paper presents a method for the identification of "edge observations" located on a boundary area constructed by a truncation variable as well as for the identification of outliers and the after fit of multivariate skew $t$-distribution(MST) to asymmetric data. The detection of edge observation is important in data analysis because it provides information on a certain critical area in observation space. The proposed method is applied to an Australian Institute of Sport(AIS) dataset that is well known for asymmetry in data space.
\end{abstract}

Keywords: Multivariate skew $t$-distribution, edge observation, outlier, ECM algorithm.

\section{1. 서론}

Azzalini (1985) 및 Azzalini와 Dalla-Valle (1996)에 의해 MSN(multivariate skew normal distribution)이 소개된 이후 비대칭 자료를 적합하기 위한 모형개발 노력이 계속되고 있다. 최근에는 MSN을 특수한 경우로서 포함하는 $\mathrm{MST}$ (multivariate skew $t$-distribution) 분포의 개발에 집중되고 있다. 정 규분포모형을 적합한 후 이상치들(outliers)을 식별하는 것은 중요한 작업으로 여겨져왔다. 물론 비대칭 자료를 위한 MST 모형의 적합 후에도 이상치의 식별은 여전히 중요하지만, 추가로 관심을 가져야할 관 측치들이 있다. 그것들은 $\mathrm{MST}$ 의 어떤 임계영역에 위치한 관측치들이다. 이들은 $\mathrm{MST}$ 의 밀도가 큰 부 분에 위치하고 있음에도 이 관측치들이 이루는 경계 밖으로는 관측치들이 거의 발생할 수 없음을 알려주 는 중요한 역할을 한다. 이러한 이유로 본 논문에서는 이들을 "에지 관측치(edge observations)" 라 부 를 것이다. 본 연구에서는 이상치 검출과 아울러 에지 관측치 식별을 위한 방법을 제공할 것이다.

$\mathrm{Sahu}$ 등 (2003)은 특별한 모수계의 한 구성원으로서 MST를 소개하였다. 이들의 MST는 Lin (2010)에 의해 혼합모형으로 확장되었다. 그리고 Pyne 등 (2009)은 Sahu 등 (2003)의 MST의 제약 버전을 소 개하였다. 최근 Lo 등 (2008)과 Lo와 Gottardo (2012)는 Bickel과 Docksum (1981)의 확장된 Box$\mathrm{Cox}$ 변환함수를 바탕으로하는 MST이 소개되기도 하였다. 그러나 본 연구에서는 Lachos 등 (2010) 및 Cabral 등 (2012)에서 사용한 SNI 족(skew-normal/independent family)의 구성원으로서의 MST를 사 용할 것이다.

${ }^{1}$ Professor, Department of Data and Information, Sangji University, 660 Woosan-Dong, Wonju, KangWonDo 220-702, Korea. E-mail: sgukim@sangji.ac.kr 
다음 절에서는 SNI-MST를 소개하고 EM 알고리즘에 의한 적합방법을 간략히 제공한다. 3 절에서는 이 상치와 에지 관측치 검출을 위한 이론적 원리를 제공한다. 4 절에서는 AIS 자료를 이용하여 제안된 검출 법의 실효성을 보인다. 5 절에서는 결론을 정리하고 몇가지 사안에 대한 토론을 제시하였다.

\section{MST에 대한 검토}

\subsection{MST 모형}

$j$ 번째 임의표본인 $p$-변량 벡터 $\boldsymbol{Y}_{j}=\left(Y_{1 j}, \ldots, Y_{p j}\right)^{T}$ 가

$$
f\left(\boldsymbol{y}_{j} ; \boldsymbol{\mu}, \boldsymbol{\Sigma}, \boldsymbol{\delta}, \nu\right)=2 t_{p}\left(\boldsymbol{y}_{j} ; \boldsymbol{\mu}, \boldsymbol{\Sigma}, \nu\right) T\left(r_{j} \sqrt{\frac{\nu+p}{\nu+D_{j}}} ; \nu+p\right), \quad j=1, \ldots, n
$$

와 같은 확률밀도를 가질 때, MST 분포를 따른다고 정의한다. 그리고 $\boldsymbol{Y}_{j} \sim \mathrm{ST}_{p}(\boldsymbol{\mu}, \boldsymbol{\Sigma}, \boldsymbol{\delta}, \nu)$ 으로 표 시하자. 여기서 $t_{p}(\cdot ; \boldsymbol{\mu}, \boldsymbol{\Sigma}, \nu)$ 는 위치모수와 척도모수가 $(\boldsymbol{\mu}, \boldsymbol{\Sigma})$ 이며, 자유도가 $\nu$ 인 $p$-변량 $t$-밀도이며, $T(\cdot ; d f)$ 는 자유도가 $d f$ 인 표준 단변량 $t$-분포함수를 나타낸다. 그리고 $D_{j}=D\left(\boldsymbol{y}_{j} ; \boldsymbol{\mu}, \boldsymbol{\Sigma}\right)=\left(\boldsymbol{y}_{j}-\right.$ $\boldsymbol{\mu})^{T} \boldsymbol{\Sigma}^{-1}\left(\boldsymbol{y}_{j}-\boldsymbol{\mu}\right)$ 으로서 Mahalanobis 거리제곱을 나타내며, $r_{j}=r\left(\boldsymbol{y}_{j} ; \boldsymbol{\mu}, \boldsymbol{\Sigma}, \boldsymbol{\delta}\right)=\boldsymbol{\delta}^{T} \boldsymbol{\Sigma}^{-1 / 2}\left(\boldsymbol{y}_{j}-\boldsymbol{\mu}\right)$ 를 나타내며, $\boldsymbol{\delta}=\left(\delta_{1}, \ldots, \delta_{p}\right)^{T}$ 은 밀도의 치우침(skewness) 모수벡터를 나타낸다. 그리고 $\boldsymbol{\Theta}$ 는 모든 (중 복되지 않는) 모수를 포함하는 벡터를 나타내기로하자.

식 (2.1)의 $\mathrm{MST}$ 는 만약 $\nu \rightarrow \infty$ 이면 $\mathrm{MSN}$ 이 되며, $\boldsymbol{\delta} \rightarrow \boldsymbol{0}$ 이면 다변량 $t$-분포 밀도가 되고, 두 조건이 동시에 만족하면 다변량 정규분포 밀도로 축소되는 매우 신축적인 모형이다.

한편, 우도함수를 알기 쉽게 나타내기 위해 식 (2.1)에서 $\boldsymbol{\delta}^{*}=\boldsymbol{\Sigma}^{1 / 2} \boldsymbol{\delta} / \sqrt{1+\boldsymbol{\delta}^{T} \boldsymbol{\delta}}$ 및 $\boldsymbol{\Omega}=\boldsymbol{\Sigma}-\boldsymbol{\delta}^{*} \boldsymbol{\delta}^{* T}$ 와 같은 재모수화가 필요한데, 이것은 언제든지

$$
\boldsymbol{\delta}=\frac{\left(\boldsymbol{\Omega}+\boldsymbol{\delta}^{*} \boldsymbol{\delta}^{* T}\right)^{-\frac{1}{2}} \boldsymbol{\delta}^{*}}{\left[1-\boldsymbol{\delta}^{* T}\left(\boldsymbol{\Omega}+\boldsymbol{\delta}^{*} \boldsymbol{\delta}^{* T}\right)^{-\frac{1}{2}} \boldsymbol{\delta}^{*}\right]^{\frac{1}{2}}}, \quad \boldsymbol{\Sigma}=\boldsymbol{\Omega}+\boldsymbol{\delta}^{*} \boldsymbol{\delta}^{* T}
$$

와 같이 되돌릴 수 있다.

\subsection{MST의 재표현 및 해석}

$U_{j} \sim \operatorname{gamma}(\nu / 2, \nu / 2)$ 를 따른다고 할 때,

$$
\left(\begin{array}{c}
Z_{j} \\
\boldsymbol{Y}_{j}
\end{array}\right) \mid U_{j}=u_{j} \sim N_{1+p}\left(\left(\begin{array}{c}
0 \\
\boldsymbol{\mu}
\end{array}\right), \frac{1}{u_{j}}\left(\begin{array}{cc}
1 & \boldsymbol{\delta}^{*} \\
\boldsymbol{\delta}^{*^{T}} & \boldsymbol{\Sigma}
\end{array}\right)\right)
$$

을 따른다고 하자. 이때 조건부 변량 $\boldsymbol{Y}_{j} \mid\left(Z_{j}>0, U_{j}=u_{j}\right)$ 은 식 (2.1)의 밀도를 가지는 것으로 알려져 있다. 여기서 $\operatorname{gamma}(\alpha, \beta)$ 은 평균이 $\alpha / \beta$ 인 감마분포를 나타낸다.

식 (2.3)을 잠시 살펴보면 다음과 같은 해석이 가능하다. 즉, $p$-변량 $\boldsymbol{Y}_{j}$ 와 공분산이 $\operatorname{cov}\left(X_{j}, \boldsymbol{Y}_{j}\right)=$ $\boldsymbol{\delta}^{*}$ 인 (즉 상관계수가 $\boldsymbol{\Sigma}^{-1 / 2} \boldsymbol{\delta}^{*}$ 인) 어떤 단변량 확률변수 $Z_{j}$ 를 고려했을 때, $Z_{j}$ 가 0 보다 큰 쪽으로 절단 된 영역에서 $\boldsymbol{Y}_{j}$ 의 밀도는 상관계수에 비례하여 치우침이 발생하게 된다. 여기서 미관측 변수 $Z_{j}$ 를 "절 단변수”라 부르자. 절단변수의 절단점은 0 으로서 분명하다. 만약 관측변수 $\boldsymbol{Y}_{j}$ 의 모든 변량이 $Z_{j}$ 와 상 관계수 1 을 가지면 $p$ 개의 각 절단점들 역시 어떤 한 점으로 분명하게 되며 대응하여 매우 큰 비대칭적 치우침이 발생하게 된다. 그러나 각각의 상관계수가 1 보다 작아지면 치우침이 약화되다가 $Z_{j}$ 가 $\boldsymbol{Y}_{j}$ 와 무상관이라면 $\boldsymbol{Y}_{j}$ 의 밀도는 치우침이 발생하지 않는다. 
식 (2.3)의 관계는 즉시 다음과 같은 위계적 구조로도 나타낼 수 있다. 즉, $X_{j} \stackrel{\text { def }}{=} Z_{j} \mid Z_{j}>0$ 이라 할 때

$$
\begin{aligned}
\boldsymbol{Y}_{j} \mid\left(X_{j}=x_{j}, U_{j}=u_{j}\right) & \sim N_{p}\left(\boldsymbol{\mu}+\boldsymbol{\delta}^{*} x_{j}, \frac{\boldsymbol{\Omega}}{u_{j}}\right) \\
X_{j} \mid U_{j}=u_{j} & \sim \operatorname{HN}\left(0, u_{j}^{-1}\right), \\
U_{j} & \sim \operatorname{gamma}\left(\frac{\nu}{2}, \frac{\nu}{2}\right),
\end{aligned}
$$

여기서 $\mathrm{HN}\left(\mu, \sigma^{2}\right)$ 는 평균과 분산이 $\left(\mu, \sigma^{2}\right)$ 인 단변량 절반정규분포(half normal distribution)를 나타 낸다.

식 $(2.4)$ 를 보면 알 수 있듯이 $u_{j}$ 와 $x_{j}$ 는 각각 관측치 $\boldsymbol{y}_{j}$ 의 산포와 평균적 위치를 결정한다. 만약 $\nu \rightarrow$ $\infty$ 이면 $U_{j}=1$ 로 퇴화되어 $\boldsymbol{Y}_{j}$ 가 정규분포를 따르도록 하지만, $\nu$ 가 작을때 $u_{j}$ 는 0 에 가까운 값을 취할 수 있는데, 이 경우 관측치 $\boldsymbol{y}_{j}$ 가 정규성에서 벗어난 관측치 즉 이상치가 된다.

한편, 식 (2.4)의 $\boldsymbol{\mu}+\boldsymbol{\delta}^{*} x_{j}$ 는 $X_{j}$ 에 대한 $\boldsymbol{Y}_{j}$ 의 회귀평면이라 할 수 있다. 이때 우리는 절단값 0에 가 까운 $x_{j}$ 에 대응하는 관측치들이 $\boldsymbol{y}_{j}$ 공간상에서 어떤 경계면을 이루게 될 것으로 기대할 수 있다. 만약 $x_{j}$ 의 예측치 $\hat{x}_{j}$ 를 구할 수 있다면, 이 값이 0 에 가까운 개체 $j$ 를 찾고 이에 대응하는 관측치 $\boldsymbol{y}_{j}$ 를 식별 할 수 있다.

이상으로부터 우리는 미관측 자료인 $u_{j}$ 와 $x_{j}$ 의 예측치를

$$
\hat{u}_{j}=E\left(U_{j} \mid \boldsymbol{y}_{j}, \widehat{\boldsymbol{\Theta}}\right) \quad \text { 및 } \quad \hat{x}_{j}=E\left(X_{j} \mid \boldsymbol{y}_{j}, \widehat{\boldsymbol{\Theta}}\right)
$$

와 같은 조건부 기대값으로 구하여 이에 대응하는 관측치가 이상치인지 그리고 에지 관측치인지를 판별 할 것이다. 그런데 이에 대한 구체적 방법은 먼저 모형 적합을 위한 $\mathrm{EM}$ 알고리즘을 소개한 후에 다시 논의하도록 하겠다.

\section{3. $\mathrm{EM}$ 알고리즘}

식 (2.4)-(2.6)의 조건부 관계로부터 완비자료 $\boldsymbol{u}=\left(u_{1}, \ldots, u_{n}\right)^{T}, \boldsymbol{x}=\left(x_{1}, \ldots, x_{n}\right)^{T}$ 및 $\boldsymbol{y}=$ $\left(\boldsymbol{y}_{1}^{T}, \ldots, \boldsymbol{y}_{n}^{T}\right)^{T}$ 의 로그-우도는

$$
\begin{aligned}
L_{c}(\boldsymbol{\Theta} \mid \boldsymbol{u}, \boldsymbol{t}, \boldsymbol{y}) \propto & \frac{1}{2} \sum_{j=1}^{n}\left\{\log |\boldsymbol{\Omega}|+u_{j}\left(\boldsymbol{y}_{j}-\boldsymbol{\mu}-\boldsymbol{\delta}^{*} x_{j}\right)^{T} \boldsymbol{\Omega}^{-1}\left(\boldsymbol{y}_{j}-\boldsymbol{\mu}-\boldsymbol{\delta}^{*} x_{j}\right)\right\} \\
& -\sum_{j=1}^{n}\left\{\log \Gamma\left(\frac{\nu}{2}\right)-\frac{\nu}{2} \log \left(\frac{\nu}{2}\right)-\frac{\nu}{2}\left(\log u_{j}-u_{j}\right)\right\}
\end{aligned}
$$

와 같이 나타낼 수 있다. 이제 $\mathrm{EM}$ 알고리즘의 $(k+1)$ 번째 단계의 $\mathrm{E}-$ 스텝에서 완비자료의 조건부 기대 값 $Q\left(\boldsymbol{\Theta} \mid \Theta^{(k)}\right)=E_{\Theta^{(k)}}\left[L_{c}(\boldsymbol{\Theta}) \mid \boldsymbol{y}\right]$ 를 계산해야 한다. 이것은 식 (2.8)를 통해 알 수 있듯이 다음과 같은 몇 가지의 충분통계량에 대한 조건부 기대값을 계산해야 하는 문제이다. 즉,

$$
\begin{aligned}
u_{j}^{(k+1)} & =E_{\boldsymbol{\Theta}^{(k)}}\left[U_{j} \mid \boldsymbol{y}_{j}\right], \\
(u x)_{j}^{(k+1)} & =E_{\boldsymbol{\Theta}^{(k)}}\left[U_{j} X_{j} \mid \boldsymbol{y}_{j}\right], \\
\left(u x^{2}\right)_{j}^{(k+1)} & =E_{\boldsymbol{\Theta}^{(k)}}\left[U_{j} X_{j}^{2} \mid \boldsymbol{y}_{j}\right]
\end{aligned}
$$

인데, 이 세 조건부 기대값은 Cabral 등 (2012)에서 명시적 형태로 주어져 있다. 이 기대값들을 바탕으 로 $\mathrm{M}$-스텝에서 모수 $Q\left(\boldsymbol{\Theta} \mid \boldsymbol{\Theta}^{(k)}\right)$ 를 최대화함으로써 추정치 $\boldsymbol{\Theta}^{(k+1)}$ 을 얻는다. 그리고 이 과정을 모수들 
이 수렴할 때까지 충분히 반복하여 최종적으로 추정치 $\widehat{\Theta}$ 를 얻게된다 (M-스텝에 대한 구체적인 과정은 Cabral 등 (2012)나 Kim (2012)를 참조하기 바란다).

$\mathrm{EM}$ 알고리즘을 수행하면 식 $(2.9)$ 으로부터 $\hat{u}_{j}$ 들을 추가적인 계산없이 얻을 수 있다. 그러나 $\hat{x}_{j}=$ $E_{\widehat{\Theta}}\left[X_{j} \mid \boldsymbol{y}_{j}\right]$ 은 $\mathrm{EM}$ 알고리즘 수행 후에 계산해야 하는데 이것에 대해서는 다음 소절에서 자세히 설명 하겠다.

2.4. $E_{\widehat{\Theta}}\left[X_{j} \mid \boldsymbol{y}_{j}\right]$ 의 계산

$\boldsymbol{Y}_{j} \sim \mathrm{ST}_{p}(\boldsymbol{\mu}, \boldsymbol{\Sigma}, \boldsymbol{\delta}, \nu)$ 일 때, 조건부 확률변수 $U_{j} \mid \boldsymbol{y}_{j}$ 는

$$
h\left(u_{j} \mid \boldsymbol{y}_{j}\right)=g\left(u ; \frac{\nu+p}{2}, \frac{\nu+D_{j}}{2}\right) \Phi\left(u_{j}^{\frac{1}{2}} r_{j}\right) T^{-1}\left(r_{j} \sqrt{\frac{\nu+p}{\nu+D_{j}}} ; \nu+p\right)
$$

의 확률밀도를 가진다 $(\mathrm{Kim}, 2012)$. 여기서 $g(u ; \alpha, \beta)$ 는 모수가 $(\alpha, \beta)$ 인 감마분포 밀도이며 $\Phi(\cdot)$ 는 $N(0,1)$ 의 분포함수를 나타내고, $r_{j}=m_{j} / V$ 인데 $m_{j}=V^{2} \boldsymbol{\delta}^{*^{T}} \boldsymbol{\Omega}^{-1}\left(\boldsymbol{y}_{j}-\boldsymbol{\mu}\right), V^{2}=\left(1+\boldsymbol{\delta}^{*^{T}} \boldsymbol{\Omega}^{-1} \boldsymbol{\delta}^{*}\right)^{-1}$ 를 각각 나타낸다. 그리고 Cabral 등 (2012)에 따르면 조건부 확률변수 $X_{j} \mid\left(\boldsymbol{y}_{j}, U_{j}=u_{j}\right)$ 는

$$
f_{x}\left(x_{j} \mid \boldsymbol{y}_{j}, u_{j}\right)=\phi\left(x_{j} ; m_{j}, u_{j}^{-1} V_{j}^{2}\right) \Phi^{-1}\left(u_{j}^{\frac{1}{2}} r_{j}\right), \quad 0<x_{j}<\infty
$$

와 같은 확률밀도를 가진다. 여기서 $\phi\left(\cdot ; \mu, \sigma^{2}\right)$ 은 $N\left(\mu, \sigma^{2}\right)$ 의 확률밀도이다. 식 (2.11)은 결국 $N\left(m_{j}\right.$, $\left.u_{j}^{-1} V_{j}^{2}\right)$ 분포의 양으로의 절단분포를 의미한다.

이때

$$
X_{j} \mid \boldsymbol{y}_{j} \sim t t_{\nu+p}\left(m_{j}, V^{2}\left[\frac{\nu+D_{j}}{\nu+p}\right] ;(0, \infty)\right)
$$

이다. 즉, 조건부 확률변수 $X_{j} \mid \boldsymbol{y}_{j}$ 은 위치모수와 척도모수가 $\left(m_{j}, V^{2}\left[\left(\nu+D_{j}\right) /(\nu+p)\right]\right)$ 이며 자유도 $\nu+p$ 인 단변량 $t$-분포의 양으로의 절단 분포를 따른다. 이에 대한 증명은 부록에 수록하였다.

이제 Kim (2008)의 Theorem 3.1 혹은 Ho 등 (2012)의 Theorem 1에 의해

$$
\hat{x}_{j}=E\left(X_{j} \mid \boldsymbol{y}_{j}, \widehat{\boldsymbol{\Theta}}\right)=\hat{m}_{j}+\hat{V}^{2} \sqrt{\frac{\hat{\nu}+\hat{D}_{j}}{\hat{\nu}+p}}\left(\frac{\hat{\nu}+p}{\hat{\nu}+p-1}\right) \times \hat{\kappa}_{j}, \quad j=1, \ldots, n
$$

단,

$$
\hat{\kappa}_{j}=\frac{1}{\sqrt{\pi(\hat{\nu}+p)}} \frac{\Gamma\left(\frac{\hat{\nu}+p+1}{2}\right)}{\Gamma\left(\frac{\hat{\nu}+p}{2}\right)} T^{-1}\left(\frac{\hat{m}_{j}}{\hat{V}} \sqrt{\frac{\hat{\nu}+p}{\hat{\nu}+\hat{D}_{j}}} ; \hat{\nu}+p\right)
$$

과 같이 얻을 수 있다.

\section{3. 실자료 예제}

AIS(Australian Institute of Sport) 자료 (Cook과 Weisberg, 1994)는 자료집단의 비대칭성 때문에 치우친 분포 모형의 적합능력을 평가하는 벤치마킹 사례로 자주 이용된다. 이 자료는 100 명의 여성과 102 명의 남성 운동선수들의 11 가지의 특성을 조사한 자료로서 우리는 이 중에 시각적 판정이 용이하도 


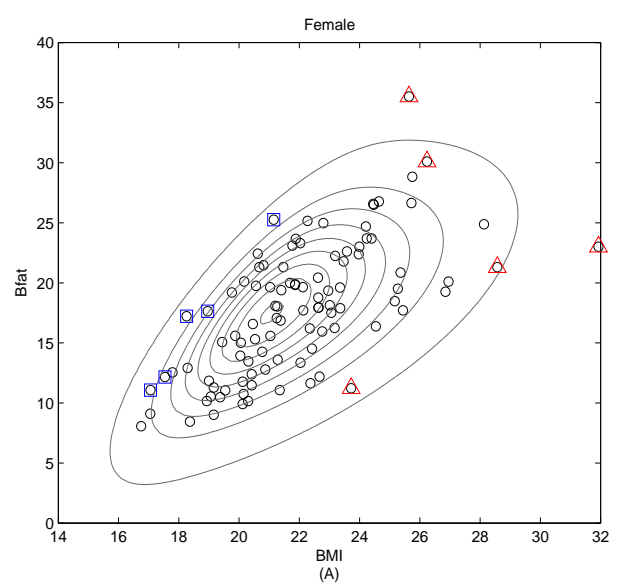

(a) Female

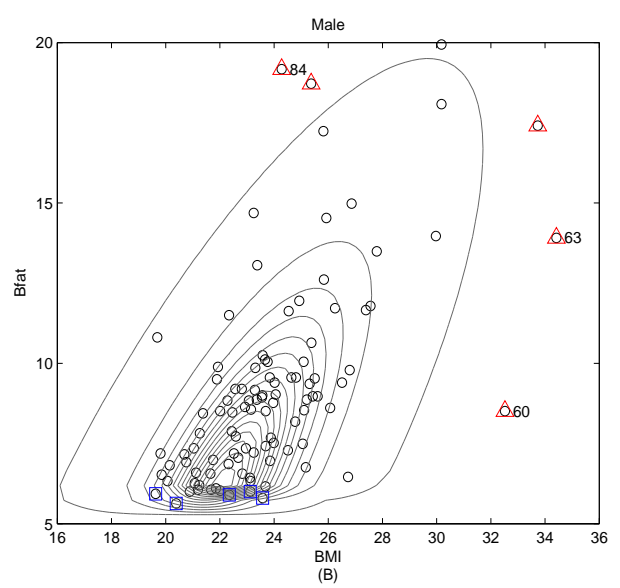

(b) Male

Figure 3.1. Results of Identification of Outliers and Edge Observations (<Symbols $>$ black $\bigcirc$ : observations, red $\triangle$ : outlying observations, blue $\square$ : edge observations, contour: fitted density).

Table 3.1. Estimates of parameters and $\hat{u}_{j}$ and $\hat{x}_{j}$ for a few observations

\begin{tabular}{cccccccccc}
\hline & Parameters & $\mu_{1}$ & $\mu_{2}$ & $\omega_{1}^{2}$ & $\omega_{12}$ & $\omega_{2}^{2}$ & $\nu$ & $\delta_{1}$ & $\delta_{2}$ \\
\hline \multirow{5}{*}{ Female } & Estimates & 19.56 & 16.98 & 3.11 & 7.94 & 28.39 & 75.30 & 2.92 & -0.11 \\
\cline { 2 - 9 } & Outlying Obs. & 75 & 56 & 70 & 53 & 63 & & & \\
& $\hat{u}_{j}$ & 0.87 & 0.89 & 0.94 & 0.95 & 0.95 & & \\
& Edge Obs. & 50 & 94 & 5 & 86 & 37 & & & \\
\hline \multirow{5}{*}{ Male } & $\hat{x}_{j}$ & 0.16 & 0.18 & 0.19 & 0.19 & 0.20 & & & \\
& Estimates & 21.82 & 5.78 & 2.22 & 0.08 & 0.02 & 3.63 & 8.80 & 23.00 \\
\cline { 2 - 9 } & Outlying Obs. & 60 & 63 & 84 & 78 & 82 & & & \\
& $\hat{u}_{j}$ & 0.13 & 0.14 & 0.17 & 0.20 & 0.22 & & & \\
& Edge Obs. & 42 & 56 & 72 & 69 & 55 & & & \\
& $\hat{x}_{j}$ & 0.03 & 0.03 & 0.05 & 0.06 & 0.08 & & \\
\hline
\end{tabular}

록 두 가지 특성변량 $\mathrm{BMI}\left(\right.$ body mass-index: $Y_{1}$ )과 Bfat(percentage of body fat: $Y_{2}$ )만을 사용할 것 이다.

Figure 3.1에 여성 집단 (a)과 남성 집단 (b) 자료들을 산점도(심볼 $\bigcirc$ )로 나타내었다. 두 집단 모두 치 우침을 가지는 비대칭성을 보여주고 있는데, 등고선으로 표현된 2-변량 $\mathrm{MST}$ 분포로 잘 적합되고 있다 (참고로 여성집단과 남성집단에 대한 적합모형의 로그-우도는 각각 -514.1394 및 -441.4471 였다). 그 리고 Table 3.1 에 두 집단에 대한 MST 모형의 모수 추정치들과 함께 가장 작은 5 개의 $\hat{u}_{j}$ 와 $\hat{x}_{j}$ 그리고 그에 대응하는 관측치의 번호를 제공하였다.

\section{1. 이상치 진단}

먼저 여성집단의 경우 자유도 추정치 $\hat{\nu}=75.30$ 로서 추정된 분포가 $t$-분포라기보다는 정규분포에 더 가 깝다. 대다수 $\hat{u}_{j}$ 들이 거의 1 에 가까운 값을 나타내었는데, 그 중 가장 작은 5 개의 관측치의 $\hat{u}_{j}$ 들은 각 각 $0.87,0.89,0.94,0.95,0.95$ 였다. 이는 0 보다는 1 에 가까운 값으로서 정규성을 넘어서는 이상치들이 
라고 말할 수 없으며, 다만 대부분의 자료보다 상대적으로 중심에서 조금 멀리 떨어져 있는 자료임을 말 해 주고 있는 것이다. 이것들은 Figure $3.1(\mathrm{a})$ 에서 빨간 $\triangle$ 로 표시되어 있다.

반면 남성집단의 경우 가장 작은 5 개의 $\hat{u}_{j}$ 는 각각 $0.13,0.14,0.17,0.20,0.22$ 을 나타내었다 (Figure $3.1(\mathrm{~b})$ 의 빨간 $\triangle$ ). 특히 처음 $60,63,84$ 번 관측치는 $t$-분포가 아닌 정규분포 하에서는 발생할 가능성이 거의 없는 것들로서 분명하게 이상치라고 판정할 수 있을 것이다. 실제로 남성집단의 자유도 추정치는 $\hat{\nu}=3.63$ 로서 추정된 밀도는 매우 두꺼운 꼬리를 가지고 있는 $t$-분포임을 말하고 있다.

\section{2. 에지 관측치 진단}

여성집단과 남성집단에 대해 가장 작은 5 개의 $\hat{x}_{j}$ 를 Table 3.1 에 수록하였다. 그리고 이에 대응하는 관측치들을 Figure 3.1(a)-(b)에 파랑 $\square$ 로 표시하였다. 이 관측치들은 일정한 경계를 형성하는데 적 합 등고선이 압축되어 나타난 임계영역과 아주 잘 일치하고 있음을 보여주고 있다. 특히 여성집단보 다 남성집단의 $\hat{x}_{j}$ 가 훨씬 작은데, 이것은 남성집단의 비대칭성이 훨씬 더 심하다는 것을 의미한다. 실 제로 Table 3.1에서 치우침 모수 추정치 $\left(\hat{\delta}_{1}, \hat{\delta}_{2}\right)$ 가 여성집단의 경우 $(2.92,-0.11)$ 인 반면 남성집단은 $(8.80,23.00)$ 로서 매우 크다는 것이 이를 확인해 주고 있다.

마지막으로 본 논문의 주제와는 다소 괴리가 있지만 흥미로운 관심거리 하나를 추가로 제공하면서 논 문을 마치도록 하겠다. 미지의 절단 전 확률변수 $Z$ 와 관측변수 $\left(Y_{1}, Y_{2}\right)$ 의 상관계수 추정치는 $\hat{\boldsymbol{\rho}}=$ $\hat{\boldsymbol{\Sigma}}^{-1 / 2} \hat{\boldsymbol{\delta}}^{*}$ 로서 얻을 수 있는데, 그 결과는 다음과 같았다. 즉, 미지의 절단변수 $Z$ 는 여성 $\mathrm{BMI}$ 와 거

\begin{tabular}{cccc}
\hline Group & BMI $\left(Y_{1}\right)$ & Bfat $\left(Y_{2}\right)$ \\
\hline Female & $Z$ & 0.95 & -0.03 \\
Male & & 0.36 & 0.93 \\
\hline
\end{tabular}

의 절대적인 상관성이 있고 여성 Bfat와는 무관한 변수이며, 반대로 남성 Bfat과는 매우 큰 상관성이 있 으며 남성 $\mathrm{BMI}$ 와는 다소 무관한 변수로 나타났다. 이러한 변수 $Z$ 가 절단이 전제됨으로써 (BMI, Bfat) 자료의 비대칭성(즉 skewness)을 유발하고 있는 것이다. 스포츠 생리학자라면 아마 변수 $Z$ 가 무엇인지 금방 알지도 모른다.

\section{4. 결론 및 토의}

본 논문에서는 비대칭 자료에 대한 MST 모형적합 후 이상치 검출과 "에지 관측치"를 식별하는 방법을 제안하였다. 이들은 관측자료 $\boldsymbol{y}_{j}$ 에 대한 미관측 자료의 조건부 기대값 $E\left(U_{j} \mid \boldsymbol{y}_{j}\right)$ 및 $E\left(X_{j} \mid \boldsymbol{y}_{j}\right)$ 으로 각 각 예측한 후 이들이 0 에 가까운지 검토하는 것이다.

본 논문에서 제공한 이상치 검출방법은 McLachlan과 Peel (2000)에서 $t$-분포 적합에 관해 소개되었던 방법이다. 다만 본 논문에서는 skew $t$-분포 적합에도 이 방법이 유효함을 보인 것이다. 그러나 저자가 아는 한 에지 관측치 개념은 본 논문에서 처음 언급한 것으로서 방법 또한 처음일 것이라 사료된다. 비 대칭 자료의 적합에 대한 관심이 증가할수록 에지 관측치 식별에 대한 관심도 함께 커질 것으로 기대된 다.

이울러 제안된 에지 관측치 식별 기법은 $\mathrm{MST}$ 혼합모형으로 확장하여 시도해 볼 수 있을 것이다. 이때 군집화된 그룹에서 식별된 에지 관측치들은 각 군집의 특성을 밝히는데 중요한 역할을 할 것으로 예상된 다. 이 문제는 본 연구의 향후 과제이다. 


\section{부록}

식 (2.12)의 중명: 식 (2.12)를 증명하기 전에 먼저 다음 성질을 알아두자.

Lemma A.1 $U \sim \operatorname{gamma}(\alpha, \beta)$ 를 따를 때, 임의의 $p$-차원 벡터 $\boldsymbol{x}$ 에 대해

$$
E\left[\phi(\boldsymbol{x} \sqrt{U} ; \mathbf{0}, \boldsymbol{\Sigma}) U^{\frac{p}{2}}\right]=t_{2 \alpha}\left(\boldsymbol{x} \sqrt{\frac{\alpha}{\beta}} ; \mathbf{0}, \boldsymbol{\Sigma}\right)\left(\frac{\alpha}{\beta}\right)^{\frac{p}{2}}
$$

이다.

위 결과는 $\operatorname{Lin}(2010)$ 의 Proposition 1에 주어진 등식의 양변을 $\boldsymbol{x}$ 에 관해 미분하면 즉시 얻을 수 있다. 이제 관측의 첨자 $j$ 를 떼고 $r=m / V$ 라 쓰면서,

$$
\begin{aligned}
f_{x}(x \mid \boldsymbol{y}) & =\int_{0}^{\infty} f(x, u \mid \boldsymbol{y}) d u=\int_{0}^{\infty} f_{x}(x \mid \boldsymbol{y}, u) h(u \mid \boldsymbol{y}) d u \\
& =T^{-1}\left(r \sqrt{\frac{\nu+p}{\nu+D}} ; \nu+p\right) \int_{0}^{\infty} \phi\left(x ; m, u^{-1} V^{2}\right) g\left(u ; \frac{\nu+p}{2}, \frac{\nu+D}{2}\right) d u \\
& =T^{-1}\left(r \sqrt{\frac{\nu+p}{\nu+D}} ; \nu+p\right) \int_{0}^{\infty} u^{\frac{1}{2} 2} \phi\left(u^{\frac{1}{2}}(x-m) ; 0, V^{2}\right) g\left(u ; \frac{\nu+p}{2}, \frac{\nu+D}{2}\right) d u \\
& =T^{-1}\left(r \sqrt{\frac{\nu+p}{\nu+D}} ; \nu+p\right) E\left[\phi\left(U^{\frac{1}{2}}(x-m) ; 0, V^{2}\right) U^{\frac{1}{2}}\right]
\end{aligned}
$$

을 얻을 수 있다. 여기서 $p=1$ 인 경우에 대하여 Lemma A.1을 이용하면,

$$
\begin{aligned}
f_{x}(x \mid \boldsymbol{y}) & =T^{-1}\left(r \sqrt{\frac{\nu+p}{\nu+D}} ; \nu+p\right) t_{\nu+p}\left((x-m) \sqrt{\frac{\nu+p}{\nu+D}} ; 0, V^{2}\right)\left(\frac{\nu+p}{\nu+D}\right)^{\frac{1}{2}} \\
& =T^{-1}\left(r \sqrt{\frac{\nu+p}{\nu+D}} ; \nu+p\right) t_{\nu+p}\left(x ; m, V^{2}\left[\frac{\nu+D}{\nu+p}\right]\right), \quad 0<x<\infty
\end{aligned}
$$

을 얻는다. 그런데

$$
\int_{0}^{\infty} t_{\nu+p}\left(x ; m, V^{2}\left[\frac{\nu+D}{\nu+p}\right]\right) d x=T\left(r \sqrt{\frac{\nu+p}{\nu+D}} ; \nu+p\right)
$$

이므로, $f_{x}(x \mid \boldsymbol{y})$ 은 $t t_{\nu+p}\left(m_{j}, V^{2}[(\nu+D) /(\nu+p)] ;(0, \infty)\right)$ 의 확률밀도임을 알 수 있다.

\section{References}

Azzalini, A. (1985). A class of distribution which includes the normal ones, Scandinavian Journal of Statistics, 33, 561-574.

Azzalini, A. and Dalla-Valle, A. (1996). The multivariate skew normal distribution, Biometrika, 83, 715-726.

Bickel, P. J. and Doksum, K. A. (1981). An analysis of transformations revisited, Journal of American Statistical Association, 76(374), 296-311.

Cabral, C. S., Lachos, V. H. and Prates, M. O. (2012). Multivariate mixture modeling using skew-normal independent distribution, Computational Statistics and Data Analysis, 56, 126-142.

Cook, R. D. and Weisberg, S. (1994). An Introduction to Regression Graphics, 56, Wiley, New York.

Ho, H. J., Lin, T. I., Chen, H.-Y. and Wang, W.-L. (2012). Some results on the truncated multivariate $t$ distribution, Journal of Statistical Planning \& Inference, 142, 25-40.

Kim, H. J. (2008). Moments of truncated Student-t distribution, Journal of Korean Statistical Society, 37, 81-87. 
Kim, S.-G. (2012). ECM Algorithm for fitting of mixtures of multivariate Skew $t$-Distribution, Communications of the Korean Statistical Society, 19, 673-684.

Lachos, V. H., Ghosh, P. and Arellano-Valle, R. B. (2010). Likelihood based inference for skew-normal independent linear mixed model, Statistica Sinica, 20, 303-322.

Lin, T.-I. (2010). Robust mixture modeling using multivariate skew $t$ distributions, Statistics and Computing, 20, 343-356.

Lo, K., Brinkman, R. R. and Gottardo, R. (2008). Automated gating of flow cytometry data via robust model-based clustering. Cytometry Part A, 73, 321-332.

Lo, K. and Gottardo, R. (2012). Flexible mixture modeling via the multivariate $t$ distribution with the Box-Cox transformation: An alternative to the skew-t distribution, Statistics and Computing, 22, $33-52$.

McLachlan, G. J. and Peel, D. (2000). Finite Mixture Models, Wiley, New York.

Pyne, S., Hu, X., Wang, K., Rossin, E., Lin, T. I., Maier, L., Baecher-Allan, C., McLachlan, G. J., Tamayo, P., Hafler, D. A., De Jager, P. L. and Mesirov, J. P. (2009). Automated high-dimensional flow cytometric data analysis, Proceedings of the National Academy of Sciences of the United States of America, 106, 8519-8524.

Sahu, S. K., Dey, D. K. and Branco, M. D. (2003). A new class of multivariate skew distribution with application to Bayesian regression model, The Canadian Journal of Statistics, 31, 129-150. 